\title{
iccafd
}

\section{DURACIÓN DE SUEÑO EN PERSONAS MAYORES CON SÍNDROME METABÓLICO}

\section{SLEEP DURATION IN OLDER PEOPLE WITH METABOLIC SYNDROME}

\author{
Pérez-López, J. 1,2 \\ Benavente-Marín, JC. ${ }^{2,3}$ \\ Wärnberg, J. 1,2 \\ Pérez-López, D. ${ }^{4}$ \\ Barón-López, FJ. 2,3 \\ Pérez-Farinós, N. ${ }^{2,3}$ \\ 1 Departamento de Enfermería. Facultad de Ciencias de la Salud. Universidad de Málaga. \\ España.jessicaperezlopez@uma.es \\ 2 Instituto de Investigación Biomédica de Málaga (IBIMA). España. \\ ${ }^{3}$ Departamento de Salud Pública y Psiquiatría. Facultad de Medicina. Universidad de Málaga. \\ España. napoleon.perez@uma.es \\ ${ }^{4}$ Instituto Educación Secundaria San Felipe Neri. Martos. España.
}

Código UNESCO: 321200 Salud Pública

Clasificación Consejo de Europa: 17. Otras: Calidad de vida

Correspondencia:

Pérez-Farinós, $\mathbf{N}$.

napoleon.perez@uma.es

DOI: http://dx.doi.org/10.24310/riccafd.2018.v7i2.5096

\section{RESUMEN}

El sueño es un indicador de la calidad de vida. Sus alteraciones pueden afectar la salud. El objetivo del estudio fue analizar el patrón de sueño en personas mayores con síndrome metabólico. Se realizó un estudio transversal en 326 participantes del nodo 01 del Estudio PREDIMEDPLUS (Universidad de Málaga). Las horas de sueño se cuantificaron de forma objetiva con un acelerómetro, y de manera subjetiva, a partir de un cuestionario. La duración media del sueño (horas) medida con el acelerómetro fue mayor en mujeres $(6,6 \pm 0,1)$ que en hombres $(6,2 \pm 0,1)$; estos datos difieren de los auto-declarados de 6,7 $\pm 0,1$ horas en hombres y $7,3 \pm 0,1$ en mujeres. Un $43 \%$ de hombres y un $30 \%$ de mujeres duermen menos de 6 horas. Un alto porcentaje de los participantes tienen un sueño deficiente. Además, se necesitan futuras investigaciones metodológicas de la evaluación del sueño.

Palabras clave: sueño, acelerómetro, cuestionario, PREDIMEDPLUS. 


\section{ABSTRACT}

Sleep is a quality-of-life indicator. Sleep alterations have health consequences. The objective of the study was to analyze the sleep pattern in elderly people with metabolic syndrome. A cross-sectional study was carried out on a sample of 326 participants of node 01 of the PREDIMEDPLUS study (University of Malaga). The hours of sleep were quantified objectively with an accelerometer, and subjectively, with a self-reported questionnaire. The average duration of sleep (hours) measured with the accelerometer was higher in women $(6.6 \pm 0.1)$ than in men $(6.2 \pm 0.1)$; these data differ from self-reported data of $6.7 \mathrm{~h} \pm 0.1$ hours in men and $7.3 \mathrm{~h} \pm 0.1$ in women. $43 \%$ of men and $30 \%$ of women sleep less than 6 hours. A high percentage of participants have a poor sleep. In addition, future methodological investigations of sleep assessment are needed.

Keywords: sleep, accelerometer, questionnaire, PREDIMEDPLUS.

\section{INTRODUCCIÓN}

El sueño forma parte fundamental de la vida de las personas y constituye una necesidad biológica que permite restablecer las funciones físicas y psicológicas básicas para alcanzar un rendimiento satisfactorio. Sus trastornos se encuentran entre los problemas de salud no declarados más importantes, con consecuencias sobre la salud y la calidad de vida de quien lo padece ${ }^{1}$.

El insomnio es el trastorno del sueño más frecuente y uno de los que mayor trascendencia sanitaria y social tiene. El paciente con sueño deficiente se queja principalmente de su insatisfacción con la calidad y/o cantidad del sueño. Esta insatisfacción puede provenir de la dificultad que tiene para quedarse dormido o para mantener el sueño a lo largo de la noche, así como del número de despertares precoces que sufre, o del sueño no reparador ${ }^{2}$.

Conocer la epidemiología del insomnio ayuda a entender la relevancia clínica de este trastorno. A pesar de los pocos datos existentes acerca de la prevalencia del insomnio en España, estos similares a los de otros países de nuestro entorno, y no existen datos sobre su incidencia.

Hay pocos estudios sobre el sueño en personas mayores, pero en nuestro ámbito alrededor de un $32 \%$ de los mayores presentan perturbaciones en la esfera del sueño, y en torno a un $12 \%$ cuando se refieren a las clasificaciones del insomnio ${ }^{3,4}$. Hay evidencias de que los trastornos del sueño contribuyen significativamente a aumentar los costes de atención de la salud. Los cambios de estilo de vida relacionados con la jubilación, la mayor incidencia de problemas de salud y el mayor uso de medicamentos sitúan a esta población en un mayor riesgo de alteraciones del sueño ${ }^{5}$.

Algunos estudios han puesto de manifiesto que el insomnio es más frecuente en las mujeres, hasta 1,5 veces más que en los hombres ${ }^{6}$. Debe tenerse en 
cuenta que la modificación en la estructura y el patrón del sueño aparece fisiológicamente con la edad y no debe confundirse con un trastorno del sueño.

La mayor parte de los estudios establecen una asociación de un patrón de sueño corto, menos de 7 horas, y largo, más de 7 horas, con la enfermedad cardiovascular y el accidente cerebrovascular ${ }^{7}$. Otro estudio afirma una relación significativa entre esta división por horas y el riesgo de padecer depresión ${ }^{8}$.

El estudio PREDIMEDPLUS es un ensayo de campo aleatorizado y multicéntrico dirigido a la prevención primaria de enfermedad cardiovascular en adultos con sobrepeso u obesidad con alto riesgo cardiovascular, mediante una intervención intensiva basada en una dieta mediterránea hipocalórica, promoción de actividad física y terapia conductual (grupo intervención), en comparación con un programa no intensivo de educación sobre dieta mediterránea para la prevención de morbimortalidad cardiovascular según los principios del ensayo PREDIMED ${ }^{9}$, junto a los cuidados habituales de los profesionales de Atención Primaria de salud, sin restricción calórica ni objetivos de pérdida de peso ni promoción de actividad física (grupo control).

El objetivo de este trabajo consiste en estudiar la duración del sueño en personas mayores con síndrome metabólico, participantes del estudio PREDIMEDPLUS, cuantificándolas mediante dos mediciones, una objetiva, como es el acelerómetro, y otra subjetiva, a partir de un cuestionario basal auto-declarado en una entrevista cara a cara con los individuos de la muestra.

\section{MATERIAL Y MÉTODOS}

Se realizó un estudio trasversal donde se analizaron los datos recogidos en la visita basal del ensayo clínico aleatorizado PREDIMEDPLUS, pertenecientes al nodo reclutador 01 de la Universidad de Málaga.

La muestra de estudio está constituida por 326 personas con alto riesgo cardiovascular, pero sin previa enfermedad cardiovascular, con edades comprendidas entre los 55 y 75 años en varones y 60 y 75 años en mujeres, con sobrepeso u obesidad (IMC $\geq 27 \mathrm{y}<40 \mathrm{~kg} / \mathrm{m}^{2}$ ) y síndrome metabólico ${ }^{10}$ (con 3 o más factores de riesgo de los siguientes: hipertensión arterial, diabetes mellitus tipo II, obesidad central y dislipidemia). Estos participantes fueron reclutados en los Centros de Salud (CS) de Atención Primaria Arroyo de la Miel (Benalmádena), Torrequebrada (Benalmádena) y Los Boliches(Fuengirola), entre abril de 2014 y noviembre de 2016.

La participación en este estudio fue voluntaria, con previa aceptación del consentimiento informado. Más detalles sobre la descripción del estudio PREDIMEDPLUS está disponible en la página web http://predimedplus.com/. El ensayo se registró el 24 de julio de 2014 en el International Standard Randomized Controlled Trial con el número 89898870. 
Los parámetros relacionados con el sueño necesarios para la realización del presente estudio fueron recogidos en la visita basal, en consulta individual. Para la medición de las horas de sueño, se utilizó una pregunta dentro de un cuestionario auto-reportado por el participante: “¿Cuántas horas duerme al día?”. Se indicó a los participantes que contaran tanto horas nocturnas como diurnas, y se contestara con valores aproximados al alza a números enteros, por lo que la mitad de una hora contaría como una hora completa al ser redondeada.

Para la medición objetiva de las horas de sueño se utilizó un acelerómetro de muñeca entregado al participante en la visita basal. Los acelerómetros registran las actividades cotidianas realizadas durante el día y los hábitos nocturnos, y permiten evaluar el patrón de sueño, el sedentarismo y la actividad física ${ }^{11,12}$.

A los participantes se les pidieron llevar un acelerómetro triaxial (GENEActiv, Activinsights Ltd, Kimbolton, Cambs, Reino Unido, http://www.geneactiv.org/) en la muñeca de la mano no dominante durante 7 días, las 24 horas del día, para tener representados tanto los días entre semana como los de fines de semana. Se entregó a los participantes una guía explicativa con preguntas y respuestas sobre el correcto funcionamiento y manejo del acelerómetro. La adherencia al protocolo es buena, ya que el acelerómetro es ergonómico, impermeable y fácil de llevar, similar a un reloj. Los problemas encontrados por los participantes para llevar el acelerómetro son mínimos, alergias a plástico o metal o un viaje programado. Tras una semana, el acelerómetro se devolvió al investigador.

Los datos obtenidos de los acelerómetros GENEActiv fueron volcados en una base de datos gestionada por el nodo del estudio PREDIMEDPLUS de la Universidad de Málaga. Para la extracción de los datos de sueño del acelerómetro se utilizó el método validado de esta publicación "Novel, Open Access Method to Assess Sleep Duration Using a Wrist-Worn Accelerometer"13, que refleja las fases donde el individuo está dormido no solo en la baja actividad del acelerómetro, sino que este permanece en una posición prácticamente constante, sin cambios de ángulos de orientación superiores a $5^{\circ}$ durante 5 minutos o más en la fase de vigilia. Además se generó automáticamente un informe personalizado para cada persona evaluada.

Se describieron las variables cuantitativas del estudio mediante el cálculo de estadísticos descriptivos media y desviación típica, y para las variables categóricas se utilizaron sus porcentajes. Se compararon las variables de estudio entre hombres y mujeres. Para las variables cuantitativas se empleó la prueba de la t de Student para muestras independientes, y para las variables cualitativas se empleó la prueba de la Chi cuadrado. El análisis de datos se ha realizado con el paquete estadístico SPSS, en su versión 21.0 para Windows.

\section{RESULTADOS}

De los 326 participantes, 165 (50,61\%) eran hombres y 161 (49,39\%) mujeres. 


\begin{tabular}{|c|c|c|c|c|c|}
\hline \multirow[b]{2}{*}{ Variable } & \multicolumn{2}{|c|}{ Hombres } & \multicolumn{3}{|c|}{ Mujeres } \\
\hline & $\mathbf{n}$ & Media (DE) & $\mathbf{N}$ & Media (DE) & $\mathbf{P}$ \\
\hline Sueño (horas) ${ }^{a}$ & 163 & $7,3 \pm 0,1$ & 160 & $6,7 \pm 0,1$ & $<0,001^{*}$ \\
\hline Sueño-acelerómetro (horas) & 113 & $6,2 \pm 0,1$ & 98 & $6,6 \pm 0,1$ & $0,016^{*}$ \\
\hline Edad (años) & 165 & $64,0 \pm 0,4$ & 161 & $65,6 \pm 0,3$ & $0,002^{*}$ \\
\hline Peso (kg) & 165 & $90,5 \pm 0,9$ & 161 & $78,2 \pm 0,8$ & $<0,001^{*}$ \\
\hline Talla (cm) & 165 & $167,7 \pm 0,5$ & 161 & $155,4 \pm 0,4$ & $<0,001^{*}$ \\
\hline $\mathrm{IMC}\left(\mathrm{kg} / \mathrm{m}^{2}\right)$ & 165 & $32,2 \pm 0,3$ & 161 & $32,3 \pm 0,3$ & 0,678 \\
\hline P17(score 1-17) & 165 & $8,9 \pm 0,2$ & 161 & $9,8 \pm 0,2$ & $0,001^{*}$ \\
\hline Diabético \% (n) & 66 & 40,2 & 59 & 36,6 & 0,280 \\
\hline Sedentario (>7 horas/día) & 49 & 29,9 & 34 & 21,1 & 0,092 \\
\hline Fumador \% (n) & 34 & 20,7 & 22 & 13,7 & $<0,001^{*}$ \\
\hline Casado/a \% (n) & 86 & 76,8 & 66 & 56,9 & --- \\
\hline Viudo/a \% (n) & 2 & 1,8 & 27 & 23,3 & \\
\hline $\begin{array}{l}\text { Soltero/a,separado/a, divorciado/a } \\
\% \text { (n) }\end{array}$ & 24 & 21,4 & 23 & 19,8 & \\
\hline Escuela primaria \% (n) & 42 & 37,5 & 56 & 48,3 & \\
\hline Escuela secundaria \% (n) & 44 & 39,3 & 40 & 34,5 & \\
\hline Estudios universitarios \% (n) & 25 & 22,3 & 18 & 15,5 & \\
\hline Está trabajando \% (n) & 31 & 27,7 & 10 & 8,6 & --- \\
\hline Ama de casa \% (n) & 1 & 0,9 & 52 & 44,8 & \\
\hline Jubilado/a \% (n) & 62 & 55,4 & 43 & 37,1 & \\
\hline Diagnóstico depresión \% (n) & 10 & 8,9 & 51 & 44,0 & $<0,001^{*}$ \\
\hline Medicaciónc \% (n) & 19 & 17,0 & 49 & 42,2 & \\
\hline
\end{tabular}

a “Cuántas horas duerme al día?”

b P17(1-17 ítems) Cuestionario de Adherencia a la Dieta Mediterránea: Puntúa sobre 17 puntos, siendo la mejor puntuación el valor más alto.

'Medicación tomada durante el último mes: tranquilizantes, sedantes, pastillas para la ansiedad, pastillas para dormir.

$p$, valor $p$ de significación estadística para la diferencia entre hombres y mujeres.

$\mathrm{DE}$, desviación estándar.

Tabla 1. Descripción de las variables de estudio. Comparación entre hombres y mujeres.

En la tabla 1 se muestran las variables de horas de sueño procedentes del cuestionario auto-reportado y de horas de sueño obtenidas del acelerómetro. En ambas se observan diferencias estadísticamente significativas entre hombres y mujeres. El sueño auto-reportado es menor en mujeres, mientras que los datos obtenidos por el acelerómetro indican un sueño mayor en ellas; y, por el contrario, el sueño auto-reportado en hombres es mayor que el indicado por el acelerómetro. En cuanto al diagnóstico de depresión, es significativamente mayor en mujeres, representando un $44 \%$ frente al $8,9 \%$ de hombres. 


\begin{tabular}{|c|c|c|c|c|c|}
\hline & Variable & $\begin{array}{l}\text { Sueño }<6 \\
\text { horas }\end{array}$ & $\begin{array}{l}\text { Sueño 6-8 } \\
\text { horas }\end{array}$ & $\begin{array}{l}\text { Sueño }>8 \\
\text { horas }\end{array}$ & $\mathbf{p}$ \\
\hline \multirow{12}{*}{ 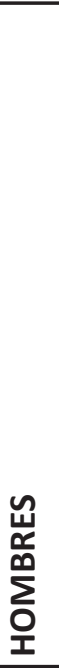 } & Porcentaje (n) & $42,5(48)$ & $54,9(62)$ & $2,6(3)$ & \\
\hline & $\begin{array}{l}\text { Sueño-acelerómetro (ho- } \\
\text { ras) }\end{array}$ & $5,2 \pm 0,1$ & $6,9 \pm 0,1$ & $8,2 \pm 0,0$ & $<0,001^{*}$ \\
\hline & Sueño (horas) & $7,0 \pm 0,2$ & $7,5 \pm 0,1$ & $8,3 \pm 0,8$ & $0,015^{*}$ \\
\hline & Edad (años) & $63,2 \pm 0,8$ & $64,1 \pm 0,7$ & $72,0 \pm 1,4$ & $0,017^{*}$ \\
\hline & IMC (kg/m²) & $32,2 \pm 0,5$ & $32,3 \pm 0,4$ & $30,0 \pm 1,3$ & 0,504 \\
\hline & P17 (score 1-17) & $8,9 \pm 0,4$ & $8,5 \pm 0,4$ & $9,3 \pm 1,5$ & 0,684 \\
\hline & Diabético \% (n) & $45,8(22)$ & $38,7(24)$ & $66,7(2)$ & -- \\
\hline & $\begin{array}{l}\text { Sedentario (>7 horas/día) } \\
\%(n)\end{array}$ & $43,8(21)$ & $29,0(18)$ & $0,0(0)$ & 0,121 \\
\hline & Fumador \% (n) & $83,3(40)$ & $72,6(45)$ & $100,0(3)$ & --- \\
\hline & Casado \% (n) & $79,2(38)$ & $74,2(46)$ & $100,0(3)$ & -- \\
\hline & Viudo \% (n) & $2,1(1)$ & $3,2(2)$ & $0,0(0)$ & \\
\hline & Depresión \% (n) & $10,4(5)$ & $6,5(4)$ & $0,0(0)$ & 0,655 \\
\hline \multirow{12}{*}{ 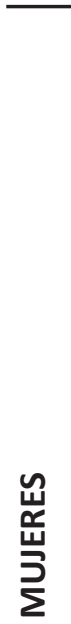 } & Porcentaje (n) & $29,6(29)$ & $64,3(63)$ & $6,1(6)$ & \\
\hline & $\begin{array}{l}\text { Sueño-acelerómetro (ho- } \\
\text { ras) }\end{array}$ & $5,4 \pm 0,1$ & $6,9 \pm 0,1$ & $8,6 \pm 0,2$ & $<0,001^{*}$ \\
\hline & Sueño (horas) & $6,6 \pm 0,2$ & $6,9 \pm 0,2$ & $6,5 \pm 0,5$ & 0,564 \\
\hline & Edad (años) & $65,3 \pm 0,8$ & $66,0 \pm 0,5$ & $66,7 \pm 2,1$ & 0,619 \\
\hline & IMC (kg/m²) & $32,8 \pm 0,8$ & $32,9 \pm 0,4$ & $31,7 \pm 1,0$ & 0,734 \\
\hline & P17 (score 1-17) & $8,8 \pm 0,5$ & $9,9 \pm 0,3$ & $11,2 \pm 0,8$ & 0,065 \\
\hline & Diabética \% (n) & $48,3(14)$ & $38,1(24)$ & $33,3(2)$ & -- \\
\hline & Sedentaria (>7 horas/día) & $20,7(6)$ & $23,8(15)$ & $16,7(1)$ & 0,890 \\
\hline & Fumadora \% (n) & $34,5(10)$ & $30,2(19)$ & $50,0(3)$ & -- \\
\hline & Casada \% (n) & $55,2(16)$ & $55,6(35)$ & $50,0(3)$ & -- \\
\hline & Viuda \% (n) & $24,1(7)$ & $22,2(14)$ & $33,3(2)$ & \\
\hline & Depresión \% (n) & $48,3(14)$ & $38,1(24)$ & $50,0(3)$ & 0,600 \\
\hline
\end{tabular}

Tabla 2, Variables de estudio según las horas de sueño (acelerómetro) según el sexo.

En la tabla 2 se describen, en cada sexo, las variables de estudio según los intervalos de sueño medidos con el acelerómetro, que comprenden: menos de 6 horas de sueño (sueño deficiente), entre 6 y 8 horas de sueño y, por último, más de 8 horas. La medición con acelerómetro no se emplea con la totalidad de la muestra, sino en un subconjunto con un total de 211 participantes. Según los datos obtenidos del acelerómetro, el $43 \%$ de hombres y el $30 \%$ de las mujeres duermen menos de 6 horas. Además, las mujeres que según el acelerómetro duermen más de 8 horas dicen dormir menos, sin llegar observarse diferencias estadísticamente significativas.

\section{DISCUSIÓN}

El insomnio constituye un problema de salud pública que afecta a millones de personas en los países industrializados, aunque su prevalencia varía ampliamente según los criterios y sistemas de clasificación que se utilicen ${ }^{14}$. Estudios 
concluyen que no es posible definir el sueño normal de manera aceptable para distintas personas y poblaciones, dado que la percepción sobre lo que cada uno considera normal en cuanto a cantidad y calidad de sueño es muy variable ${ }^{15}$.

En los participantes estudiados, las horas de sueño auto-reportado por los participantes del estudio que duermen menos de 6 horas está sobreestimado en comparación con los datos del acelerómetro, esta diferencia se observa sobre todo en los hombres que duermen menos de 6 horas.

Además, en el rango de mujeres que duermen más de 8 horas ocurre justo a la inversa, según los datos del acelerómetro duermen más de lo que dicen dormir.

Según algunos estudios las mujeres sufren más insomnio que los hombres ${ }^{3}$. Estos datos no coinciden con nuestros resultados, lo que puede explicarse porque que utilizan cuestionarios auto-reportados para cuantificar el sueño.

Para el estudio del sueño se deben tener en cuenta multitud de factores que pueden afectar y sesgar los resultados. El sueño en horas auto-reportado por el participante es menos fiable para medir la duración real del sueño al depender de la información subjetiva; de igual forma el sueño obtenido mediante el acelerómetro también presenta inconvenientes, ya que el algoritmo para detectar el comienzo del sueño expresado/definido como periodo de inactividad sostenida basados en la ausencia de cambio en el ángulo del brazo de $5^{\circ}$, puede no siempre detectarse correctamente.

\section{CONCLUSIONES}

Los resultados obtenidos en este estudio en participantes mayores con síndrome metabólico muestran que un $42 \%$ de hombres y el $30 \%$ de las mujeres duermen menos de 6 horas según el acelerómetro.

Comparando la estimación de la duración del sueño con acelerómetro y el sueño auto-reportado, el sueño auto-reportado de las mujeres es menor que los datos obtenidos por el acelerómetro; y, por el contrario, el sueño auto-reportado en hombres es mayor que el indicado por el acelerómetro.

Los métodos de estimación de los patrones de sueño requieren algoritmos precisos y podrían beneficiarse del complemento de un registro de sueño detallado, incluyendo preguntas específicas como hora de acostarse, hora de dormirse, despertares nocturnos, hora de levantarse, siesta, etc.

En este estudio sólo se analiza la cantidad (horas de sueño), pero se debería estudiar el impacto del sueño sobre determinantes de salud utilizando otras variables como calidad de vida, realizando preguntas sobre calidad de sueño, fatiga, somnolencia diurna, síntomas de ansiedad y/o depresión, nivel de atención, concentración y memoria y calidad del trabajo, entre otros. 


\section{REFERENCIAS}

1. Artiach G, Del Cura M,I, Díaz del Campo P, et al. Guía de práctica clínica para el manejo de pacientes con insomnio en atención primaria. Madrid: Ministerio de Ciencia e Innovación; 2009.

2. Cappuccio FP, Miller MA. Sleep and cardio-metabolic disease. Curr Cardiol Rep [Internet], 2017 Sep 19 [citado 21 Mar 2018];19(11):110, doi: 10,1007/s11886-017-09160 .

3. Vela-Bueno, A, De Iceta M, Fernandez, C. Prevalence of sleep disorders in Madrid, Spain. Gac Sanit. 1999 Nov-Dic;13(6):441-8.

4. Blanco J, Mateos R. Prevalencia de trastornos del sueño en el anciano. Interpsiquis. 2005; 36:1457-64.

5. Montgomery P, Dennis J. Bright light therapy for sleep problems in adults aged $60+$, Cochrane Database of Systematic Reviews 2002;(2):CD003403.

6. López de Castro, F, Fernández O, Mareque MA. Abordaje terapeútico del insomnio. Semergen. 2012;38(4):233-240.

7. Ge B, Guo X. Short and long sleep durations are both associated with increased risk of stroke: a meta-analysis of observational studies. Int J Stroke. 2015 Feb;10(2):17784, doi: 10,1111/ijs, 12398 .

8. Zhai L, Zhang $H$, Zhang D. Sleep duration and depression among adults: A meta-analysis of prospective studies. Depress Anxiety. 2015 Sep;32(9):664-70, doi: $10,1002 / \mathrm{da}, 22386$.

9. Estruch R, Ros E, Salas-Salvadó J, Covas MI, Corella D, Arós F, Martínez-González MA. Primary prevention of cardiovascular disease with a mediterranean diet. N Engl J of Med. 2013 Abr 4;368(14):1279-90. doi: 10,1056/NEJMoa1200303,.

10. Alberti K G, Eckel R H, Grundy SM, Zimmet PZ, Cleeman JI, Donato KA, et al. Harmonizing the Metabolic Syndrome: A Joint Interim Statement of the International Diabetes Federation Task Force on Epidemiology and Prevention; National Heart, Lung, and Blood Institute; American Heart Association; World Heart Federation; International Atherosclerosis Society; and International Association for the Study of Obesity. Circulation. 2009 Oct 20;120(16):1640-5. doi: 10.1161/CIRCULATIONAHA.109.192644.

11. Wärnberg J, Moreno-Morales N, Pérez-López J, Benavente-Marín JC, FernándezGarcía JC, Pérez-Farinós N, et al. Estudio de acelerometría en el ensayo PREDIMEDPLUS; patrones de actividad física, sedentarismo y sueño. En: Actividad Física para mayores, Investigación, enseñanza y práctica. Málaga, España: Delegación de Deportes, Juventud y Educación de la Diputación de Málaga; 2017;94-102.

12. Santos-Lozano A, Garatachea N. Tendencias actuales de la acelerometría para la cuantificación de la actividad física. Revista Iberoamericana de Ciencias de la Actividad Física y el Deporte, 2012;1(1):24-32. Disponible en: http://www.riccafd.uma.es/ DOCUMENTOS/articulos/VOL001/N1/Accelerometria.pdf.

13. Vincent T, Sabia S, Anderson KN, Dentol SJ, Oliver J, Catt M, et al. A novel, open access method to assess sleep duration using a wrist-worn accelerometer. Plos ONE. 2015 Nov 16;10(11). doi:10.137/journnal.pone.0142533.

14. Nacional Institute for clinical excellence. Guiance on the use of zaleplon, zolpidem and zopiclona for the short-term management of insomnia. Technology apprai-sal 
guidance. 2004 Apr 28; Disponible en: http://wwwnice.org.uk/nicemedia/pdf/TA077fullguidance.pdf

15. Alberdi Sudupe J, Castro Dono C, Pérez Ramírez L, Fernández Díaz A. Insomnio primario. Guías Clínicas Fisterra. 2011[citado 2018 Mar 21]. Disponible en: http:// www.fisterra.com/guias-clinicas/insomnio-primario/

Referencias totales citadas: 15

Referencias citadas correspondientes a la Rev Ib CC Act Dep: 1 